Original article

\title{
Endothelial progenitor cells: Are they displaying a function in autoimmune disorders?
}

\author{
Angelo Ferrante ${ }^{\mathrm{a}, 1}$, Giuliana Guggino ${ }^{\mathrm{a}, *, 1}$, Diana Di Liberto ${ }^{\mathrm{b}}$, Francesco Ciccia ${ }^{\mathrm{a}}$, \\ Paola Cipriani ${ }^{c}$, Carmela Rita Balistreri ${ }^{b}$, Guido Sireci ${ }^{b}$, Roberto Giacomellic ${ }^{\text {, }}$ \\ Giovanni Triolo ${ }^{\mathrm{a}}$ \\ a Dipartimento di Medicina Interna e Specialistica, Sezione di Reumatologia, Università degli Studi di Palermo, Italy \\ ${ }^{\mathrm{b}}$ Dipartimento di Biopatologia e Biotecnologie Mediche, Università degli Studi di Palermo, Italy \\ ' Dipartimento di Biotecnologie e Scienza Clinica Applicata, Divisione di Reumatologia, Scuola di Medicina, Università degli Studi dell'Aquila, Italy
}

\section{A R T I C L E I N F O}

\section{Article history:}

Received 16 December 2015

Received in revised form 27 April 2016

Accepted 1 May 2016

Available online 3 May 2016

\section{Keywords:}

EPCs

Rheumatoid arthritis

LES

Vasculitis

\begin{abstract}
A B S T R A C T
Endothelial Progenitor Cells (EPCs) are bone marrow derived cells able to differentiate in mature endothelial cells (EC) contributing to the generation of new vessels, connecting to fibronectin, and forming colonies and/or colony forming units.

Since circulating EPCs can be actively considered part of endothelial damage in several cardiovascular diseases and autoimmune disorders the possibility to have a measure for endothelium damage should be considered of interest to predict the patient out-come. At the same time the EPCs proliferative and regenerative role could be considered for therapeutic applications.

Studies have been performed to elucidate the role of EPCs in Systemic Sclerosis and many review and articles published on this topic.

In the present paper we aimed to review the role of EPCs in other autoimmune disorders.
\end{abstract}

(c) 2016 Published by Elsevier Ireland Ltd.

\section{Introduction}

Endothelial Progenitor Cells (EPCs) are bone marrow derived cells able to differentiate in mature endothelial cells (EC) contributing to the generation of new vessels, connecting to fibronectin, and forming colonies and/or colony forming units (Asahara, 1997). The phenotypical marker of EPCs is CD133 that is absent on mature endothelial cells. Other surface markers were identified on EPCs, $\mathrm{CD}^{+} 4^{+} / \mathrm{VEGFR}^{+} / \mathrm{CD} 146^{+}$(Rauscher et al., 2003). However, to date there are no standardized methods to quantify and identify EPCs and the protocols used vary between studies (Kuwana and Okazaki, 2012). In particular, studies on quantification of circulating EPCs number in various autoimmune diseases are controversial probably because of different microeviroment, drugs, cytokine and other factors modifying their survival (Cipriani et al., 2011; Kuwana et al., 2004; Del Papa et al., 2004; Paleolog et al., 2005; Moonen et al., 2007).

\footnotetext{
* Corresponding author.

E-mail address: Giuliana.guggino@unipa.it (G. Guggino).

1 The first two authors contributed equally to this work.
}

The number of circulating EPCs depends on integrity of vessel wall and on their capability to migrate (Cipriani et al., 2011; Hill, 2003). These cells show high proliferation capability and are referred as "true or classical" EPC (Cipriani et al., 2011; Hristov and Weber, 2008). More recently, studies have also described another subset of EPCs (named monocytic derived EPCs) with lower ability to create new vessels in vivo than "classical" EPCs (Urbich and Dimmeler, 2004). Monocytic derived EPCs were found increased in several autoimmune disorders showing paradoxically, enhanced angiogenetic but reduced vasculogenetic properties [Yamaguchi et al., 2010]. Monocytic EPCs are oligopotent cells able to differentiate not only into endothelial cells but also in pericytes and smooth muscle cells [Yamaguchi et al., 2010].

Abnormalities in circulating EPC levels and function have been observed in a large number of studies on different cardiovascular diseases (CVDs). As result, EPCs have been suggested as diagnostic and prognostic CVD biomarkers (as extensively summarized in Balistreri et al., 2015). Cotemporally, a significant correlation between in vitro EPC senescence and CVD risk profile has been also reported in donors (as extensively summarized in Balistreri et al., 2015). Thus, EPCs might be considered as an optimal predictive biomarker for vascular function and cardiovascular risk. Actually, it is also emerging that the number of circulating EPC cells studies 
might also be used as an optimal measure for endothelium damage, and consequently it should be considered of interest to predict the patient out-come. At the same time the EPCs proliferative and regenerative role could be considered for therapeutic applications, as demonstrated in several studies in animal models and clinical human trials (as reviewed in Balistreri et al., 2015).

These relevant features of circulating EPC cells in CVDs and the clinical applications which might derived from them, have also led to elucidate the role of EPCs in some autoimmune diseases, such as Systemic Sclerosis. Many review and articles have been published on this topic (Kahlenberg, 2011; Yamaguchi et al., 2010; Hill, 2003; Manetti, 2010; Blaise et al., 2014; Andrigueti et al., 2015). Here, we aimed to review the role of EPCs in rheumatic autoimmune diseases (ARDs), including systemic lupus erythematosus, vasculitis and rheumatoid arthritis.

\section{ARDs and high prevalence of CVDs}

Patients affected by ARDs show a significant increased risk for developing CVDs. The risk is approximately 1.4 fold higher in patients with Rheumatoid Arthritis (RA) (Watson et al., 2003) and up to 5-6 fold higher in patients with Systemic Lupus Erythematosus (SLE) (Manzi et al., 1997). This risk is exaggerated in younger SLE patients and may be up to 52 fold greater in those aged 35-44 years. The onset of premature CVDs in RA and SLE patients is related to a co-presence of traditional risk factors (i.e. hypertension, diabetes mellitus and dyslipidaemia) and disease- specific factors (i.e. corticosteroid use and proinflammatory cytokines) (Esdaile et al., 2001; Bruce et al., 2003; Bruce, 2005; Lee et al., 2006). Furthermore, patients with ARDs might develop subclinical CVDs, which are also increased in patients with RA and SLE. Carotid artery plaque, carotid intima-medial thickness (IMT) and coronary calcification are more prevalent in both RA and SLE (Ferrante, 2009; Kumeda, 2002; Asanuma, 2003; Roman et al., 2003; Roman et al., 2006; Ahmad et al., 2007; Wang et al., 2009).

As above mentioned, CVDs in ARDs seem to be caused by traditional and non- traditional risk factors. Besides other factors, inflammation and immunologic abnormalities, the quantity and quality of lipoproteins, hypertension, insulin resistance/hyperglycemia, obesity and underweight, presence of platelets bearing complement protein $\mathrm{C} 4 \mathrm{~d}$, reduced number and function of endothelial progenitor cells, apoptosis of endothelial cells, epigenetic mechanisms, renal disease, periodontal disease, depression, hyperuricemia, hypothyroidism, sleep apnea and vitamin D deficiency may contribute to the premature CVD. Although most research has focused on systemic inflammation, vascular inflammation may play a crucial role in the premature CVD in ARDs. It may be involved in the development and destabilization of both atherosclerotic lesions and of aortic aneurysms (a known complication of ARDs). Inflammation in subintimal vascular and perivascular layers appears to frequently occur in CVD, with a higher frequency in ARD than in non-ARD patients. It is possible that this inflammation is caused by infections and/or autoimmunity, which might have consequences for treatment. Importantly, drugs targeting immunologic factors participating in the subintimal inflammation (e.g., T- and B-cells) might have a protective effect on CVD. Interestingly, vasa vasorum and cardiovascular adipose tissue may play an important role in atherogenesis. Inflammation and complement depositions in the vessel wall are likely to contribute to vascular stiffness. Based on biopsy findings, also inflammation in the myocardium and small vessels may contribute to premature CVD in ARDs (cardiac ischemia and heart failure) (Castellon and Bogdanova, 2016) There is an enormous need for an improved CVD prevention in ARDs. Studies examining the effect of
DMARDs/biologics on vascular inflammation and CV risk are warranted.

\section{Endothelium dysfunction in ARD patients}

It is well known that endothelial dysfunction represents the early stage of the development of CVD. In RA, endothelial dysfunction occurs early in the disease course and is associated with systemic inflammation (Vaudo et al., 2004; Kerekes et al., 2008; Hannawi et al., 2009). The pathophysiological mechanisms which result in endothelial dysfunction in these patients are, however, unknown, although associations have been found between abnormal flow medial dilation and low-density lipoprotein (Vaudo et al., 2004), rheumatoid factor (Marder et al., 2011), anticyclic

citrullinated peptide (APCA) antibodies (Hjeltnes et al., 2011) and interferon-gamma (Kerekes et al., 2008) in RA. In patients with SLE, FMD has been associated with elevated interferon-alpha (Somers et al., 2012), lupus disease activity, and altered serum plasminogen activator inhibitor-1 (PA-1) (Somers et al., 2005).

\section{An imbalance between endothelial damage and repair: a possible cause of high prevalence of CVDs in ARD patients}

The endothelium, in common with other tissues, is in a constant state of wear and repair. In the post-embryonic state, new vessels were thought to develop only from the proliferation of mature endothelial cells. This process is termed angiogenesis and is important in tissue repair following physical injury or ischaemia. In aged individuals, or in patients affected by chronic inflammatory diseases, such as ARDs, an imbalance between EC damage and impaired EC repair has been suggested as proposed initial step in the development of atherosclerosis, or of other pathological agerelated vascular conditions, i.e. medial degeneration and vascular remodeling associated with other CVDs. Early detection of cellular and molecular mechanisms related to such imbalance with potential therapeutic interventions, may reduce atherogenesis and subsequent cardiovascular events, and onset and progression of medial degeneration and vascular remodeling related of other agerelated CVDs (Williamson et al., 2012; Felice et al., 2013; Olivieri et al., 2013). Endogenous regeneration of the vessel wall involves a large panel of cells, such as EPCs and mature EC cells, which are capable to restore the endothelium in a concerted interaction. Thus, it is important to understand the cellular and molecular mechanisms of EPCs and their role in ARDs.

In the next paragraphs, we tried to give a contribution describing the role of EPC in SLE, vasculitis and RA.

\subsection{EPC in SLE}

Evidence from epidemiological studies demonstrates that SLE is associated with an increased risk of premature cardiovascular complications due to accelerated atherosclerosis, which significantly contributes to morbidity and mortality in these patients. Different studies suggest that traditional risk factors may play a role but do not fully explain the increased prevalence of atherosclerosis (Petri et al., 1992; Rahman et al., 1999; Esdaile et al., 2001; Rajagopalan et al., 2004). In addition the so-called non-traditional factors, such as systemic chronic inflammation, the presence of autoantibodies and the use of immunosuppressive drugs, have been suggested to have a role (Asanuma, 2003; Schoenfeld, 2013).

It has been previously reported that SLE is characterized by accelerated endothelial cell (EC) apoptosis, which correlates with reduced endothelial function, and that circulating EC can be used as a marker of vessel damage (Asahara, 1997). A crucial aspect to preserve endothelial integrity is to maintain the balance between 
endothelial damage and repair. Vascular repair appears to be mediated by the recruitment of bone marrow-derived EPCs to the site of endothelial injury (Peichev et al., 2000). The EPCs are a heterogeneous population that contribute to the restoration of endothelial function because of their ability to differentiate into mature ECs once incorporated into circulating blood (Deng et al., 2010). Although modulation of EPC numbers has been identified in the course of cardiovascular disease or vascular trauma (which appears to be predictive of clinical coronary events in the general population), there is still much controversy regarding their true identity and role. In SLE, while most of the studies demonstrated low percentages of circulating EPC (Lee et al., 2007; Moonen et al., 2007; Westerweel et al., 2007; Robak et al., 2009), results are inconsistent, most likely due to different protocols adopted for identifying EPC (Hooks et al., 1979). Nevertheless, whether the number of circulating EPC can predict cardiovascular events in patients with SLE remains to be answered by prospective studies. Pro-angiogenic factors, such as VEGF can mobilize EPCs and may potentiate their recruitment to the site of vascular injury (Asahara et al., 1999; Rafii et al., 2002). Several cytokines are involved in EPC mobilization, survival, recruitment or function (Mao, 2012; Musilli, 2015; Rodríguez-Carrio, 2012). Besides the formation of autoantibodies, one of the most prominent features in patients with SLE is the continuous activation of the type I interferon (IFN) system (Tsokos, 2011; Ronnblom et al., 2011; Cederblad et al., 1998). Increased levels of type I IFN in SLE sera were reported already more than 30 years ago, and with the techniques of genome-wide expression analysis, it was later shown that the majority of patients with SLE have an activated type I IFN system, as judged by the increased expression of type I IFN-induced genes (a type I IFN signature) (Chiche, 2014). The over activated type I IFN system in SLE can be, at least partly, explained by the presence of endogenous interferon inducers in such patients, which triggers the plasmacytoid dendritic cells (pDCs) to type I IFN production (Vallin et al., 1999; Rajagopalan et al., 2004). Several investigators have proposed that type I interferons (IFNs) play an important role in the pathogenesis of accelerated atherosclerosis in patients with adult-onset SLE (Denny et al., 2007; Bennett et al., 2003; Werner et al., 2005), through deleterious pleiotropic effects on the vasculature. Type I IFNs induce significant impairment of the capacity of EPCs to differentiate into mature endothelial cells and repair the vasculature (Denny et al., 2007; Thacker et al., 2012). In murine models of lupus and atherosclerosis, type I IFN blocking agents abrogates endothelial dysfunction, the prothrombotic phenotype, plaque formation, and abnormal vasculogenesis (Thacker et al., 2010; Mohan et al., 2015; Kahlenberg, 2011). In addition type I IFNs repress expression of the pro-angiogenic cytokine IL- $1 \beta(41)$. Importantly, type I IFNs have also been shown to upregulate NLRP3, caspase-1 and IL18 in human EPC cultures, which results in enhanced production of IL-18. IL-18, in turn, has inhibitory effects on EPC differentiation to mature endothelial cells (Kahlenberg, 2014). Inhibition of caspase- 1 in these cultures prevents the detrimental effects of type I IFNs (Kahlenberg, 2014), and absence of functional caspase-1 also renders murine bone marrow EPC cultures resistant to the detrimental effects of pristane-induced lupus and improves endothelial function in vivo (Cates et al., 2015). Finally it was shown that the negative effect of IFN on the function and differentiation of EPCs could be enhanced by the action of IL-10, a cytokine involved in the pathogenesis of SLE by promoting the production of high titer of autoantibodies via B cell activation (Jennette et al., 2013).

\subsection{EPCs in vasculitis}

Systemic vasculitis is characterised by systemic inflammation, endothelial activation, and (in some cases) necrosis of blood vessel walls leading to multiorgan injury (Hoffman and Calabrese,
2014, Savage, 2009). Endothelial activation and injury are central to the pathogenesis of vasculitides with increased endothelial cell adhesion molecule expression, and a switch to a prothrombotic endothelial phenotype, both contributing to the vascular pathology (Tomasson et al., 2009; Blake et al., 1985). In systemic vasculitis impaired endothelial repair capacity of EPC was addressed and the significant inflammatory milieu could adversely affect EPC function, resulting in defective colony formation, adhesion and ability to integrate into vascular networks. Although it has been recognized for many years that endothelial damage is central to the pathogenesis of vasculitis surprisingly little is known about the balance between endothelial injury and repair (Brogan et al., 2004; Woywodt et al., 2003; Nakatani et al., 2003).

de Groot et al. (2007) have studied the role of EPCs in ANCAassociated vasculitis. They have found not different percentage of EPCs between patients with active disease and controls, conversely EPCs count was increased in patients with disease remission respect to controls (Závada et al., 2009). Thus, EPCs could be considered as a marker of vascular regeneration inversely correlate with disease activity. In this regards EPCs could be involved in the repair of vascular defects in patients with ANCA associated vasculitis (AAV). A similar time course for EPCs has been reported in patients with Kawasaki disease where the number of EPCs was increased during the subacute phase and decreased during active phase or remission (de Groot et al., 2007).

In contrast, however recent studies showed significant reduced levels of numbers of EPC-CFU during the active phase of the disease and suggested that impairment of EPC function was mediated in part by inflammatory circulating endothelial cells (Holmén et al., 2005).

\section{3. $E P C s$ in $R A$}

Rheumatoid Arthritis (RA) is an autoimmune systemic disease characterized by cardiovascular comorbidity (del Rincon et al., 2007), mostly due to its inflammatory nature which contributes to an accelerated development of atherosclerosis (del Rincon et al., 2007; Libby et al., 2002). Atherosclerotic plaque and sinovia from RA patients share same features, including a typical inflammatory microenvironment composed by infiltrating leucocytes (Full et al., 2009), cytokines, adhesion molecules and an activated endothelial phenotype. The loss of protective antioxidant and anti-inflammatory mechanisms seems to be responsible for the endothelial dysfunction which is the earliest, but yet reversibile, stage of atherosclerotic plaque formation (Deanfieldet et al., 2007). Supporting this hypothesis, endothelial dysfunction has been found also in young RA patients without traditional cardiovascular risk factors (Hansel et al., 2003), thus suggesting the involvement of disease-related mechanisms.

Physiologically, vasculogenesis, the de novo formation of blood vessels from mesoderm, is driven by the recruitment of undifferentiated mesodermal cells to the endothelial lineage, or EPCs, and has a key role in embryo and adult tissue development, in vascular repair and also in atherosclerosis (Paleolog et al., 2005; Peichev et al., 2000; Asahara, 1997; Jodon de Villeroché et al., 2010; Pakozdi et al., 2009).

During vasculogenesis, EPCs, recruited from the bone marrow, differentiate in situ into mature endothelial cells under the influence of nitric oxide (NO) produced by the activation of the endothelial NO synthase (eNOS) (Aicher et al., 2003). Vasculogenesis require a tightly regulated activation of several growth factors and their receptors. Recruitment of EPCs to the site of endothelial injury is induced and potentiated by VEGF as well as by several proinflammatory and immunosupressor cytokines, such as IL-1 $\beta$, IL-6, IL-8, IL-17A, TGF- $\beta$, GM-CSF and IFN- $\alpha$ (Rodríguez-Carrio, 2012). Recently, it has been reported that VEGFR-1-induced proliferation 
of bone marrow hematopoietic cells promotes chronic inflammation in a rheumatoid arthritis murine model (Aicher et al., 2003), thus confirming that inhibiting VEGFR-1 activity might be therapeutically beneficial for RA patients.

An inverse correlation between EPCs number and the Framingham risk score (Hill, 2003) in both long-standing (GonzalezJuanatey et al., 2003) and early RA patients have been documented (Bergholm et al., 2002). On the other hand have been also described increased numbers of late-outgrowth EPCs in RA, on owing an increased colony formation capacity. Indeed two EPCs subpopulations, called monocytic and hemangioblastic EPCs have been recently identified as early- outgrowth and late-outgrowth EPCs, respectively (Hristov and Weber, 2008).

Finally corticosteroids and tumor necrosis factor-alpha (TNF- $\alpha$ ) blockers were described to stimulate EPCs number and function and to restore defective vasculogenesis (Grisar et al., 2007; Ablin et al., 2006). Further details, however, on the effects of these agents, specifically on the function of late-outgrowth EPCs in long-term controlled studies, are needed, in order to evaluate the effect of this class of drugs on atherosclerotic process progression.

\section{Conclusions}

In describing the role of EPCs in ARDs, we evidenced their altered number and function and additionally underlined that their modulation in vivo seem to restore rejuvenation and to improve cardiovascular outcomes. In order to achieve very efficient therapeutic applications experimental studies in humans and non-human models and ex vivo functional analysis would be performed. Furthermore, a deeper understanding of how monocytic derived EPCs and EPCs facilitate endothelial repair would lead to the identification of novel therapeutic targets across a range of patient groups, who develop early or accelerated CVDs. Specifically in patients with ARDs, and particularly in RA and SLE, it remains to be determined whether simply controlling inflammation will be sufficient to restore EPC or monocyte EPCs function. In summary, preventing premature CVD remains an important challenge in the management of patients with ARDs, and particularly with RA and SLE; where newer agents for controlling disease activity with greater efficacy, are investigated

There are no targeted therapies to reduce CVD in these patients and well established treatments in the general population (e.g. statins) have not yet conclusively shown benefit in RA or SLE. There is great potential to utilise the homeostatic endothelial repair mechanisms to prevent the development of endothelial dysfunction, at a time point long before the development of clinical disease or even appearance of atherosclerotic plaque. In order to develop and implement these novel approaches, it is imperative to study the function and role of these cells in more detail both in ARD patients and in health in order to develop targeted therapies to reverse the clear abnormalities in ECP function in ARDs.

\section{References}

Ablin, J.N., et al., 2006. Effect of anti-TNF-alpha treatment on circulating endothelial progenitor cells (EPCs) in rheumatoid arthritis. Life Sci. 79, 2364-2369.

Ahmad, Y., Shelmerdine, J., Bodill, H., Lunt, M., Pattrick, M.G., Teh, L.S., et al., 2007. Subclinical atherosclerosis in systemic lupus erythematosus (SLE): the relative contribution of classic risk factors and the lupus phenotype. Rheumatology (Oxford) 46, 983-988.

Aicher, C., et al., 2003. Essential role of endothelial nitric oxide synthase formobilization of stem and progenitor cells. Nat. Med. 9 (11), 1370-1376.

Andrigueti, F.V., Arismendi, M.I., Ebbing, P.C., Kayser, C., 2015. Decreased numbers of endothelial progenitor cells in patients in the early stages of systemic sclerosis. Microvasc. Res. 98 (March), 82-87.

Asahara, T., et al., 1999. VEGF contributes to postnatal neovascularization by mobilizing bone marrow-derived endothelial progenitor cells. EMBO J. 18, 3964-3972.
Asahara, T., 1997. Isolation of putative progenitor endothelial cells for angiogenesis. Science 2757, 9647.

Asanuma, Y., 2003. Premature coronary- artery atherosclerosis in systemic lupus erythematosus. N. Engl. J. Med. 349, 2407-2415.

Balistreri, C.R., Buffa, S., Pisano, C., Lio, D., Ruvolo, G., Mazzesi, G., 2015. Are endothelial progenitor cells the real solution for cardiovascular diseases? Focus on controversies and perspectives. Biomed Res Int. 2015, 835934, http:// dx.doi.org/10.1155/2015/835934.

Bennett, L., et al., 2003. Interferon and granulopoiesis signatures in systemic lupus erythematosus blood. J. Exp. Med. 197, 711-723.

Bergholm, M., et al., 2002. Impaired responsiveness to NO in newly diagnosed patients with rheumatoid arthritis. Arterioscler. Thromb. Vasc. Biol. 22 (10), 1637-1641.

Blaise, S., Roustit, M., Carpentier, P., Seinturier, C., Imbert, B., Cracowski, J.L., 2014 The digital thermal hyperemia pattern is associated with the onset of digital ulcerations in systemic sclerosis during 3 years of follow-up. Microvasc. Res. 94 (July), 119-122.

Blake, D.R., et al., 1985. Endothelial cell cytotoxicity in inflammatory vascular diseases-the possible role of oxidised lipoproteins. Ann. Rheum. Dis. 44, $176-182$.

Brogan, P.A., et al., 2004. Endothelial and platelet microparticles in vasculitis of the young. Arthritis Rheum. 50, 927-936.

Bruce, I.N., et al., 2003. Risk factors for coronary heart disease in women with systemic lupus erythematosus. Arthritis Rheum. 48, 3159-3167.

Bruce, I.N., 2005. 'Not only. . . but also': factors that contribute to accelerated atherosclerosis and premature coronary heart disease in systemic lupus erythematosus. Rheumatology (Oxford) 44, 1492-1502.

Castellon, X., Bogdanova, V., 2016. Chronic inflammatory diseases and endothelial dysfunction. Aging Dis. 7 (January (1)), 81-89, http://dx.doi.org/10.14336/AD. 2015.0803 (eCollection).

Cates, A.M., et al., 2015. Interleukin 10 hampers endothelial cell differentiation and enhances the effects of interferon $\alpha$ on lupus endothelial cell progenitors. Rheumatology 54 (June (6)), 1114-1123.

Cederblad, B., et al., 1998. Patients with systemic lupus erythematosus have reduced numbers of circulating natural interferon-a- producing cells. J. Autoimmun. 11, 465-470.

Chiche, L., 2014. Modular transcriptional repertoire analyses of adults with systemic lupus erythematosus reveal distinct type I and type II interferon signatures. Arthritis Rheumatol. 66, 1583-1595.

Cipriani, P., et al., 2011. Cellular players in angiogenesis during the course of systemic sclerosis. Autoimmun. Rev. 10 (10), 641-646.

Deanfieldet, J.E., et al., 2007. Endothelial function and dysfunction: testing and clinical relevance. Circulation 10, 1285-1295.

Del Papa, N., et al., 2004. Circulating endothelial cells as a marker of ongoing vascular disease in systemic sclerosis. Arthritis Rheum. 50 (4), 1296-1304.

Deng, X.L., et al., 2010. Comparative study on circulating endothelial progenitor cells in systemic lupus erythematosus patients at active stage. Rheumatol. Int. 30, 1429-1436.

Denny, M., et al., 2007. Interferon-a promotes abnormal vasculogenesis in lupus: a potential pathway for premature atherosclerosis. Blood 110, 2907-2915.

de Groot, K., et al., 2007. Vascular endothelial damage and repair in antineutrophil cytoplasmic antibody-associated vasculitis. Arthritis Rheum. 56 (November (11)), 3847-3853.

del Rincon, I., et al., 2007. Acceleration of atherosclerosis during the course of rheumatoid arthritis. Atherosclerosis 195 (2), 354-360.

Esdaile, J.M., Abrahamowicz, M., Grodzicky, T., Li, Y., Panaritis, C., Du Berger, R., et al., 2001. Traditional Framingham risk factors fail to fully account for accelerated atherosclerosis in systemic lupus erythematosus. Arthritis Rheum. 44, 2331-2337.

Felice, F., Barsotti, M.C., Poredos, P., Balbarini, A., Di Stefano, R., 2013. Effect of aging on metabolic pathways in endothelial progenitor cells. Curr. Pharm. Des. 19 (13), 2351-2365.

Ferrante, A., 2009. Long-term anti- tumour necrosis factor therapy reverses the progression of carotid intima-media thickness in female patients with active rheumatoid arthritis. Rheumatol. Int. 30, 193-198.

Full, L.E., et al., 2009. The inextricable link between atherosclerosis and prototypical inflammatory diseases rheumatoid arthritis and systemic lupus erythematosus. Arthritis Res. Ther. 11 (2), 217.

Gonzalez-Juanatey, C., et al., 2003. HLADRB1 status affects endothelial function in treated patients with rheumatoid arthritis. Am. J. Med. 114 (8), 647-652

Grisar, J., et al., 2007. Endothelial progenitor cells in active rheumatoid arthritis: effects of tumour necrosis factor and glucocorticoid therapy. Ann. Rheum. Dis. $66,1284-1288$.

Hannawi, T.H., et al., 2009. Inflammation predicts accelerated brachial arterial wall changes in patients with recent-onset rheumatoid arthritis. Arthritis Res. Ther. 11 (2), R51.

Hansel, S., et al., 2003. Endothelial dysfunction in young patients with long-term rheumatoid arthritis and low disease activity. Atherosclerosis 170, 177-180.

Hill, J.M., 2003. Circulating endothelial progenitor cells, vascular function, and cardiovascular risk. New Engl. J. Med. 348, 593-600.

Hjeltnes, G., Hollan, I., Forre, O., et al., 2011. Anti-CCP and RF IgM: predictors of impaired endothelial function in rheumatoid arthritis patients. Scand. J Rheumatol. 40, 422-427.

Hoffman, G.S., Calabrese, L.H., 2014. Vasculitis: determinants of disease patterns. Nat. Rev. Rheumatol. 10 (8), 454-462. 
Holmén, C., et al., 2005. Circulating inflammatory endothelial cells contribute to endothelial progenitor cell dysfunction in patients with vasculitis and kidney involvement. J. Am. Soc. Nephrol. 16, 3110-3120.

Hooks, J.J., et al., 1979. Immune interferon in the circulation of patients with autoimmune disease. N. Engl. J. Med. 301, 5-8.

Hristov, M., Weber, C., 2008. Endothelial progenitor cells in vascular repair and remodeling. Pharmacol. Res. 58 (2), 148-151.

Jennette, J.C., et al., 2013. 2012 revised International Chapel Hill Consensus conference nomenclature of vasculitides. Arthritis Rheum. 65 (1), 1.

Jodon de Villeroché, V., et al., 2010. Enhanced late-outgrowth circulating endothelial progenitor cell levels in rheumatoid arthritis and correlation with disease activity. Arthritis Res. Ther. 12, R27.

Kahlenberg, J.M., 2011. Inflammasome activation of IL-18 results in endothelial progenitor cell dysfunction in systemic lupus erythematosus. J. Immunol. 187 6143-6156.

Kahlenberg, J.M., 2014. An essential role for caspase-1 in the induction of murine lupus and its associated vascular damage. Arthritis Rheum. 66, 153-162.

Kerekes, G., Szekanecz, Z., Der, H., et al., 2008. Endothelial dysfunction and atherosclerosis in rheumatoid arthritis: a multiparametric analysis using imaging techniques and laboratory markers of inflammation and autoimmunity. J. Rheumatol. 35, 398-406.

Kumeda, Y., 2002. Increased thickness of the arterial intima-media detected by ultrasonography in patients with rheumatoid arthritis. Arthritis Rheum. 46, 1489-1497.

Kuwana, M., Okazaki, Y., 2012. Quantification of circulating endothelial progenitor cells in systemic sclerosis: a direct comparison of protocols. Ann. Rheum. Dis. 71, 617-620.

Kuwana, M., et al., 2004. Defective vasculogenesis in systemic sclerosis. Lancet 364, 603-610.

Lee, A.B., Godfrey, T., Rowley, K.G., et al., 2006. Traditional risk factor assessment does not capture the extent of cardiovascular risk in systemic lupus erythematosus. Intern. Med. J. 36, 237-243.

Lee, P.Y., et al., 2007. Type I interferon as a novel risk factor for endothelial progenitor cell depletion and endothelial dysfunction in systemic lupus erythematosus. Arthritis Rheum. 56, 3759-3769.

Libby, P., et al., 2002. Inflammation and atherosclerosis. Circulation 105, 1135-1143.

Manetti, M., 2010. Mechanisms in the loss of capillaries in systemic sclerosis: angiogenesis versus vasculogenesis. J. Cell. Mol. Med. 14, 1241-1254

Manzi, S., Meilahn, E.N., Rairie, J.E., et al., 1997. Age-specific incidence rates of myocardial infarction and angina in women with systemic lupus erythematosus: comparison with the Framingham study. Am. J. Epidemiol. $145,408-415$

Mao, A., 2012. Modulation of the number and functions of endothelial progenitor cells by interleukin $1 \beta$ in the peripheral blood of pigs: involvement of p38 mitogen-activated protein kinase signaling in vitro. J. Trauma Acute Care Surg. 73, 1145-1151.

Marder, W., Khalatbari, S., Myles, J.D., et al., 2011. Interleukin 17 as a novel predictor of vascular function in rheumatoid arthritis. Ann. Rheum. Dis. 70, $1550-1555$.

Mohan, S., et al., 2015. Endothelial progenitor cell phenotype and function are impaired in childhood-onset systemic lupus erythematosus. Arthritis Rheumatol. 67, 2257-2262.

Moonen, J.R., et al., 2007. Reduced number and impaired function of circulating progenitor cells in patients with systemic lupus erythematosus. Arthritis Res. Ther. 9, R84.

Musilli, C., 2015. Endothelial soluble factors mediate differentiation of circulating endothelial precursors. J. Cardiovasc. Pharmacol. 66 (August (2)), 223-227.

Nakatani, K., et al., 2003. Circulating endothelial cells in Kawasaki disease. Clin. Exp Immunol. 131, 536-540.

Olivieri, F., Recchioni, R., Marcheselli, F., et al., 2013. Cellular senescence in cardiovascular diseases: potential age-related mechanisms and implications for treatment. Curr. Pharm. Des. 19, 1710-1719.

Pakozdi, A., et al., 2009. Endothelial progenitor cells in arthritis-associated vasculogenesis and atherosclerosis. Joint Bone Spine 76, 581-583.

Paleolog, E., et al., 2005. It's all in the blood: circulating endothelial progenitor cells link synovial vascularity with cardiovascular mortality in rheumatoid arthritis? Arthritis Res. Ther. 7, 270.

Peichev, M., et al., 2000. Expression of VEGFR-2 and CD133 by circulating human CD34(+) cells identifies a population of functional endothelial precursors. Blood 95, 952-958.

Petri, M., et al., 1992. Risk factors for coronary artery disease in patients with systemic lupus erythematosus. Am. J. Med. 93, 513-519.
Rafii, S., et al., 2002. Efficient mobilization and recruitment of marrow-derived endothelial and hematopoietic stem cells by adenoviral vectors expressing angiogenic factors. Gene Ther. 9, 631e41.

Rahman, P., et al., 1999. Contribution of traditional risk factors to coronary artery disease in patients with systemic lupus erythematosus. J. Rheumatol. 26 2363-2368.

Rajagopalan, S., et al., 2004. Endothelial cell apoptosis in systemic lupus erythematosus: a common pathway for abnormal vascular function and thrombosis propensity. Blood 103, 3677-3683.

Rauscher, F.M., et al., 2003. Aging, progenitor cell exhaustion, and atherosclerosis. Circulation 108, 457-463.

Robak, E., et al., 2009. Circulating endothelial cells and angiogenic proteins in patients with systemic lupus erythematosus. Lupus 8, 332-341.

Rodríguez-Carrio, J., 2012. Circulating endothelial cells and their progenitors in systemic lupus erythematosus and early rheumatoid arthritis patients. Rheumatology (Oxford) 51, 1775-1784.

Roman, M.J., Shanker, B.A., Davis, A., et al., 2003. Prevalence and correlates of accelerated atherosclerosis in systemic lupus erythematosus. N. Engl. J. Med. 349, 2399-2406.

Roman, M.J., Moeller, E., Davis, A., et al., 2006. Preclinical carotid atherosclerosis in patients with rheumatoid arthritis. Ann. Intern. Med. 144, 249-256.

Ronnblom, L., et al., 2011. The type I interferon system in the development of lupus. Semin. Immunol. 23, 113-121.

Savage, C.O., 2009. Vascular biology and vasculitis. APMIS 127 (Suppl), 37-40.

Schoenfeld, S.R., 2013. The epidemiology of atherosclerotic cardiovascular disease among patients with SLE: a systematic review. Semin. Arthritis Rheum. 43, 77-95.

Somers, E.C., Marder, W., Kaplan, M.J., et al., 2005. Plasminogen activator inhibitor-1 is associated with impaired endothelial function in women with systemic lupus erythematosus. Ann. N. Y. Acad. Sci. 1051, 271-280.

Somers, E.C., Zhao, W., Lewis, E.E., et al., 2012. Type I interferons are associated with subclinical markers of cardiovascular disease in a cohort of systemic lupus erythematosus patients. PLoS One 7, e37000.

Thacker, S., et al., 2010. The detrimental effects of interferon-a on vasculogenesis in lupus are mediated by repression of IL-1 pathways: potential role in atherogenesis and renal vascular rarefaction. J. Immunol. 185, 4457-4469.

Thacker, S., et al., 2012. Type I interferons modulate endothelial function, repair, thrombosis and plaque severity in murine models of lupus and atherosclerosis Arthritis Rheum. 64, 2975-2985.

Tomasson, G., et al., 2009. Thromboembolic disease in vasculitis. Curr. Opin. Rheumatol. 21, 41-46.

Tsokos, G.C., 2011. Systemic lupus erythematosus. N. Engl. J. Med. 365, 2110-2121.

Urbich, C., Dimmeler, S., 2004. Endothelial progenitor cells: characterization and role in vascular biology. Circ. Res. 95, 343-353.

Vallin, H., et al., 1999. Patients with systemic lupus erythematosus (SLE) have a circulating inducer of interferon-alpha (IFN-a) production acting on leucocytes resembling immature dendritic cells. Clin. Exp. Immunol. 115, 196-202.

Vaudo, G., Marchesi, S., Gerli, R., et al., 2004. Endothelial dysfunction in young patientswith rheumatoid arthritis and lowdisease activity. Ann. Rheum. Dis. 63, 31-35.

Wang, S., Yiu, K.H., Mok, M.Y., et al., 2009. Prevalence and extent of calcification over aorta, coronary and carotid arteries in patients with rheumatoid arthritis. J. Intern. Med. 266, 445-452.

Watson, D.J., Rhodes, T., Guess, H.A., 2003. All-cause mortality and vascular events among patients with rheumatoid arthritis, osteoarthritis, or no arthritis in the UK General Practice Research Database. J. Rheumatol. 30, 1196-1202.

Werner, N., et al., 2005. Circulating endothelial progenitor cells and cardiovascular outcomes. N. Engl. J. Med. 353, 999-1007.

Westerweel, P.E., et al., 2007. Haematopoietic and endothelial progenitor cells are deficient in quiescent systemic lupus erythematosus. Ann. Rheum. Dis. 66, $865-870$.

Williamson, K., Stringer, S.E., Alexander, M.Y., 2012. Endothelial progenitor cells enter the aging arena. Front. Physiol. 20, 3.

Woywodt, A., et al., 2003. Circulating endothelial cells as markers for ANCA-associated small-vessel vasculitis. Lancet 361, 206-210.

Yamaguchi, Y., et al., 2010. Enhanced angiogenic potency of monocytic endothelial progenitor cells in patients with systemic sclerosis. Arthritis Res. Ther. 12, R205.

Závada, J., et al., 2009. Reduced number of endothelial progenitor cells is predictive of early relapse in anti-neutrophil cytoplasmic antibody-associated vasculitis. Rheumatology (Oxford) 48, 1197-1220. 